\title{
PHOTOMETRIC PHASE VARIATIONS OF LONG-PERIOD ECCENTRIC PLANETS
}

\author{
Stephen R. Kane And Dawn M. Gelino \\ NASA Exoplanet Science Institute, Caltech, MS 100-22, 770 South Wilson Avenue, Pasadena, CA 91125, USA; skane@ipac.caltech.edu \\ Received 2010 June 28; accepted 2010 September 22; published 2010 November 4
}

\begin{abstract}
The field of exoplanetary science has diversified rapidly over recent years as the field has progressed from exoplanet detection to exoplanet characterization. For those planets known to transit, the primary transit and secondary eclipse observations have a high yield of information regarding planetary structure and atmospheres. The current restriction of these information sources to short-period planets may be abated in part through refinement of orbital parameters. This allows precision targeting of transit windows and phase variations which constrain the dynamics of the orbit and the geometric albedo of the atmosphere. Here, we describe the expected phase function variations at optical wavelengths for long-period planets, particularly those in the high-eccentricity regime and multiple systems in resonant and non-coplanar orbits. We apply this to the known exoplanets and discuss detection prospects and how observations of these signatures may be optimized by refining the orbital parameters.
\end{abstract}

Key words: planetary systems - techniques: photometric - techniques: radial velocities

\section{INTRODUCTION}

The currently known diversity of exoplanets is greatly attributable to the revolution of the transit detection method over the past 10 years. The measurement of radius and hence density were the first steps from the results of this technique, but soon to follow were atmospheric studies from both primary transit and secondary eclipse. However, the unknown inclination of the planetary orbits makes this technique only applicable to a relatively small fraction of the known exoplanets. For the nontransiting planets, reflected light and phase variations present an additional avenue through which to investigate planetary atmospheres (Charbonneau et al. 1999; Leigh et al. 2003). The net result of this new information has lead to an unprecedented ability to characterize exoplanets.

Phase functions in the infrared (IR) primarily measure the thermal properties of the planet, whereas optical measurements probe the planetary albedo. The relation between giant planet atmospheres and phase curves have been described in detail by Sudarsky et al. (2005). Iro \& Deming (2010) further investigate the time variation of the atmospheres in the IR for eccentric planets using radiative transfer models. The phase variation of our own solar system has been investigated by Dyudina et al. (2005) and it has been shown that phase functions can be used to produce longitudinal thermal maps of exoplanets (Cowan \& Agol 2008). In addition, phase curves of terrestrial planets have been considered by Mallama (2009).

Phase variations of exoplanets in the IR and optical regimes have had success due to increased access to improved instrumentation and space-based observatories. This has primarily been investigated for transiting planets since the edge-on orbital plane produces the highest phase amplitude. Examples of observed phase variations in the IR (using Spitzer) include HD 189733b (Knutson et al. 2009b) and HD 149026b (Knutson et al. 2009a). Examples in the optical include Kepler observations of HAT-P-7b (Welsh et al. 2010) and phase variations detected in the light curve of CoRoT-1b (Snellen et al. 2009).

Kane \& von Braun $(2008,2009)$ showed that planets in eccentric orbits have inflated transit probabilities, as demonstrated by HD 17156b (Barbieri et al. 2007) and HD 80606b (Laughlin et al. 2009). These types of planets will produce rela- tively high phase amplitudes during a brief period (periaston passage) of the orbit. However, phase variations of non-transiting planets have been restricted to hot Jupiters, including $v$ And $\mathrm{b}$ (Harrington et al. 2006) and HD 179949b (Cowan et al. 2007). There have been searches for phase variations of, for example, $\mathrm{HD} 75289 \mathrm{Ab}$ (Rodler et al. 2008) and $\tau$ Boo b (Charbonneau et al. 1999; Rodler et al. 2010) but no signatures were detected in either case. Exploring the atmospheric properties of longerperiod planets requires taking advantage of highly eccentric non-transiting systems. Thus, we also constrain the inclination and hence the mass of such planets.

Here, we investigate the expected photometric phase amplitude of long-period eccentric planets and show how planetary orbits in resonance can result in ambiguous phase variation detections. We further apply this analysis by calculating maximum flux ratios for the known exoplanets and considering several interesting case studies. We also determine the effective orbital phase regime over which detectability is maximized and show how refinement of orbital parameters can allow efficient targeted observations at these times. This study is intended from an observers point of view in so far as these observable variations can be reconnected back to the theoretical models of exoplanetary atmospheres.

\section{EXOPLANET PHASE VARIATIONS}

In this section, we establish the theoretical framework which will be applied in the remainder of the paper, similar to the formalism used by Collier Cameron et al. (2002) and more recently by Rodler et al. (2010). Figure 1 shows a top-down view of an elliptical planetary orbit. The phase angle $\alpha$ is described by

$$
\cos \alpha=\sin (\omega+f),
$$

where $\omega$ is the argument of periastron and $f$ is the true anomaly. The phase angle is defined to be $\alpha=0^{\circ}$ when the planet is at superior conjunction ("full" phase). In terms of orbital parameters, this location in the orbit occurs when $\omega+f=270^{\circ}$.

The flux at wavelength $\lambda$ incident upon the planet is described by

$$
F_{i}(\lambda)=\frac{L_{\star}(\lambda)}{4 \pi r^{2}},
$$




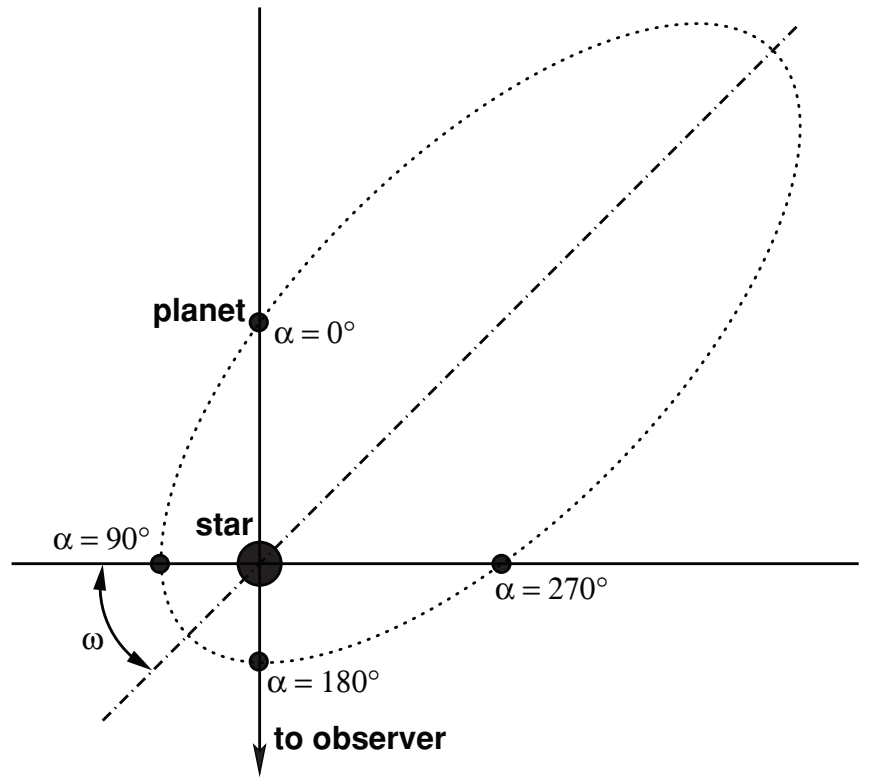

Figure 1. Orbit of eccentric planet, showing orbit phase angles corresponding to full $\left(\alpha=0^{\circ}\right)$, first quarter $\left(\alpha=90^{\circ}\right)$, new $\left(\alpha=180^{\circ}\right)$, and third quarter $\left(\alpha=270^{\circ}\right)$ phases.

where $L_{\star}$ is the luminosity of the star and $r$ is the star-planet separation. This separation is given by

$$
r=\frac{a\left(1-e^{2}\right)}{1+e \cos f},
$$

where $a$ is the semimajor axis and $e$ is the orbital eccentricity. The geometric albedo of a planet is defined at $\alpha=0^{\circ}$ as follows:

$$
A_{g}(\lambda)=\frac{F_{r}(0, \lambda)}{F_{i}(\lambda)},
$$

where $F_{r}$ is the reflected light from the planet. The planetary flux received at Earth is then

$$
f_{p}(\alpha, \lambda)=A_{g}(\lambda) g(\alpha, \lambda) F_{i}(\lambda) \frac{R_{p}^{2}}{d^{2}},
$$

where $R_{p}$ is the planetary radius, $d$ is the distance to the star, and $g(\alpha, \lambda)$ is the phase function. Since, the stellar flux received at Earth is

$$
f_{\star}(\lambda)=\frac{L_{\star}(\lambda)}{4 \pi d^{2}},
$$

then the flux ratio of the planet to the host star is defined as

$$
\epsilon(\alpha, \lambda) \equiv \frac{f_{p}(\alpha, \lambda)}{f_{\star}(\lambda)}=A_{g}(\lambda) g(\alpha, \lambda) \frac{R_{p}^{2}}{r^{2}}
$$

and thus contains three major components: the geometric albedo, the phase function, and the inverse-square relation to the star-planet separation. Note that for a circular orbit only the phase function is time-dependent.

\subsection{Wavelength Dependence}

As noted in the previous section, the observed flux ratio from an exoplanet is wavelength-dependent. In particular, the atmospheric composition drives the scattering properties and thus the forms of the geometric albedo and phase function. This dependence has been considered in great detail by Sudarsky et al. (2005) in which they construct empirical models for exoplanet atmospheres and integrate over the surface with assumed opacities depending upon atmospheric composition. This thorough analysis is not reproduced here, but we do use the results of their analysis for a restricted wavelength, particularly with regards to the albedo function discussed in the following sections. Here, we confine our study to optical wavelengths centered on $550 \mathrm{~nm}$. This broadly encompasses the results obtained by such studies by Collier Cameron et al. (2002), Leigh et al. (2003), and Rodler et al. (2010). This also places the study near the peak response of the Kepler CCD, the relevance of which will be discussed in later sections.

\subsection{Geometric Albedo}

It has been shown through atmospheric models that there is a dependence of the geometric albedo of giant planets on the semimajor axis of the orbit (Sudarsky et al. 2000, 2005; Cahoy et al. 2010). We direct the reader to Figure 9 of Sudarsky et al. (2005), which details the wavelength and star-planet separation dependence of the geometric albedo. Jupiter is known to have a visual geometric albedo of $\sim 0.52$. However, the strong irradiation of the atmospheres of giant planets in shortperiod orbits results in the removal of reflective condensates from the upper atmospheres and thus a significant lowering of the geometric albedo. Observations of HD 209458b using the Microvariability and Oscillations of STars (MOST) satellite by Rowe et al. (2008) failed to detect phase variations and thus they were able to place an upper limit of $A_{g}<0.08$, subsequently investigated using model atmospheres by Burrows et al. (2008). More recent observations of HAT-P-7b using Kepler by Welsh et al. (2010) revealed phase variations in the light curve from which they were able to deduce a geometric albedo of 0.18 .

Through the examples mentioned above, and the consideration of the theoretical models of Sudarsky et al. (2005), we have constructed a model which approximates the albedo of giant planets as a function of star-planet separation (see Equation (3)). This is a hyperbolic tangential function of the form

$$
A_{g}=\frac{\left(e^{r-1}-e^{-(r-1)}\right)}{5\left(e^{r-1}+e^{-(r-1)}\right)}+\frac{3}{10} .
$$

Equation (8) is plotted in Figure 2, showing the semimajor axes of HAT-P-7b and Jupiter for reference. This function represents well the rapid rise in optical albedo between 0.2 and $1 \mathrm{AU}$ described by Sudarsky et al. (2005) as well as the continued rise beyond $2 \mathrm{AU}$ whereby water clouds begin to be present. Though broadly robust to encompass both the theoretical calculations and the limited number of measured examples mentioned above, this empirical function does not account for planetary gravity whose variation may affect the atmospheric properties and thus the albedo properties.

\subsection{Phase Function}

A planetary phase function can be considered to be defined by the continuous presence of an imaginary line connecting the center of the star and planet which is normal to the day-night terminator of the planet. The phase function of a Lambert sphere assumes the atmosphere isotropically scatters over $2 \pi$ steradians and is described by

$$
g(\alpha, \lambda)=\frac{\sin \alpha+(\pi-\alpha) \cos \alpha}{\pi}
$$




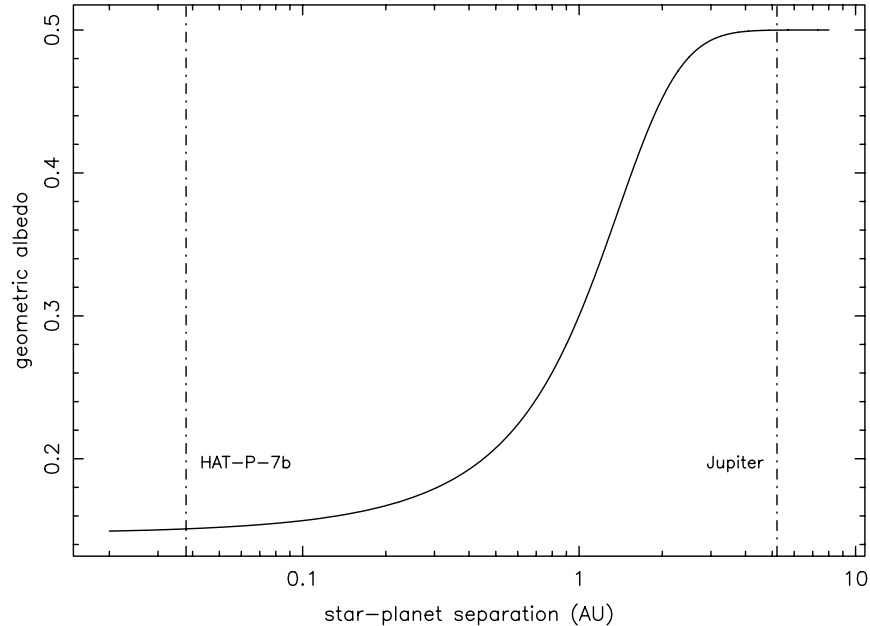

Figure 2. Approximation of the geometric albedo distribution for giant planets, where $\lambda \sim 550 \mathrm{~nm}$.

and is thus normalized to lie between 0 and 1 . For a circular orbit, the phase function applied to the flux ratio relation (Equation (7)) results in both a phase function and flux ratio which are maximum at a phase angle of zero. Generalizing the phase angle (see Equation (1)) and thus the phase function for an eccentric orbit requires first solving Kepler's equation

$$
M=E-e \sin E,
$$

where $M$ is the mean anomaly and $E$ is the eccentric anomaly. The true anomaly is then related to the eccentric anomaly by

$$
\cos f=\frac{\cos E-e}{1-e \cos E}
$$

where the true anomaly establishes the time-dependent variation of the phase function.

For the analysis performed here, we adopt the approach of Collier Cameron et al. (2002) and Rodler et al. (2010) which utilizes the empirically derived phase function of Hilton (1992). This is based upon observations of Jupiter and Venus and incorporates substantially more back-scattering due to cloudcovering. This approach contains a correction to the planetary visual magnitude of the form

$$
\Delta m(\alpha)=0.09\left(\alpha / 100^{\circ}\right)+2.39\left(\alpha / 100^{\circ}\right)^{2}-0.65\left(\alpha / 100^{\circ}\right)^{3},
$$

which leads to a phase function given by

$$
g(\alpha)=10^{-0.4 \Delta m(\alpha)},
$$

where the wavelength dependence has been removed (see Section 2.1). This Hilton phase function is used throughout the remainder of this paper.

Shown in Figure 3 are phase functions and normalized flux ratios for various eccentricities and orbital orientations. Note that the maximum flux ratio does not necessarily occur at zero phase angle for a non-circular orbit. This is because the orbital distance is changing and indeed we shall show in later sections that the star-planet separation component of Equation (7) becomes dominant for highly eccentric orbits. This time-lag between maximum flux ratio and maximum phase was also noted by Sudarsky et al. (2005).

\subsection{Orbital Inclination}

For interacting systems, many of the system parameters depend on the orbital inclination angle, $i$, of the system (for example, see Gelino et al. 2006). For exoplanetary systems, given an assumed albedo, the true amplitude of the phase variation can be used to estimate the inclination angle and therefore constrain the mass of the planet derived from radial velocity data. To add the effect of inclination angle to the phase function, the phase angle (Equation (1)) is modified as follows:

$$
\cos \alpha=\sin (\omega+f) \sin i \text {. }
$$

At first and third quarter $\left(\alpha=90^{\circ}\right.$ and $\alpha=270^{\circ}$ ), the flux ratio is completely independent of inclination angle (see Figure 1). The effect of inclination on the shape of the phase function is quite small and has negligible effect on the location of the minimum and maximum values of the flux ratio, as shown by Figure 20 of Sudarsky et al. (2005). One complicating factor in this simple inclination consideration is that any additional light sources (i.e., planets) in a given system will dilute the signature from the dominant light-reflecting planet. The dilution of the signature will remain constant if the additional planets are in face-on $\left(i=0^{\circ}\right)$ orbits. This will be discussed further in Section 3.1.

\section{MULTI-PLANET SYSTEMS}

Detection of multi-planet systems is becoming more frequent as we are increasingly able to probe into smaller mass and longer-period regimes of parameter space. If indeed single planet systems are rare, then it is highly likely that the phase curve from a particular planet will be "contaminated" by the reflected light of other planets in the system. For planets which are similar in size, the effect of an outer planet to the combined phase curve will be small since (from Equation (7)) $\epsilon(\alpha, \lambda) \propto r^{-2}$. Here we discuss the specific cases of orbital coplanarity and resonant orbits.

\subsection{Coplanarity}

Additional planets in a system serve to dilute the signature from the dominant light-reflecting planet. Depending upon planet formation scenarios, it cannot be taken for granted that planets within a system will lie in coplanar orbits. As shown in Section 2.4, the phase function may be reduced in amplitude significantly for orbits inclined relative to the line of sight, to the extreme of eliminating a time-variable photometric signature of the orbit if it is face-on $\left(i=0^{\circ}\right)$.

Consider the case of the planetary system orbiting the star $v$ And, which was first discovered by Butler et al. (1999). A search for reflected light from the innermost planet was carried out by Collier Cameron et al. (2002), but only a marginal detection was produced leaving an ambiguity of the result concerning the degeneracy between the planetary radii and assumed albedos. The $v$ And system is one of the few systems which has been monitored astrometrically as well as spectroscopically in order to provide constraints on the orbital inclinations of the planets (McArthur et al. 2010). The inclinations of the outer two planets (c and d), with semimajor axes of $0.83 \mathrm{AU}$ and $2.53 \mathrm{AU}$, were measured to be $\sim 8^{\circ}$ and $24^{\circ}$ respectively. The factor of three increase in orbital distance of the outer planet results in a factor of nine less contribution to the total planetary reflected light from the system. However, the relative inclinations of the planets cause the phase function of the outer planet to be almost three times stronger than that of the inner planet. 

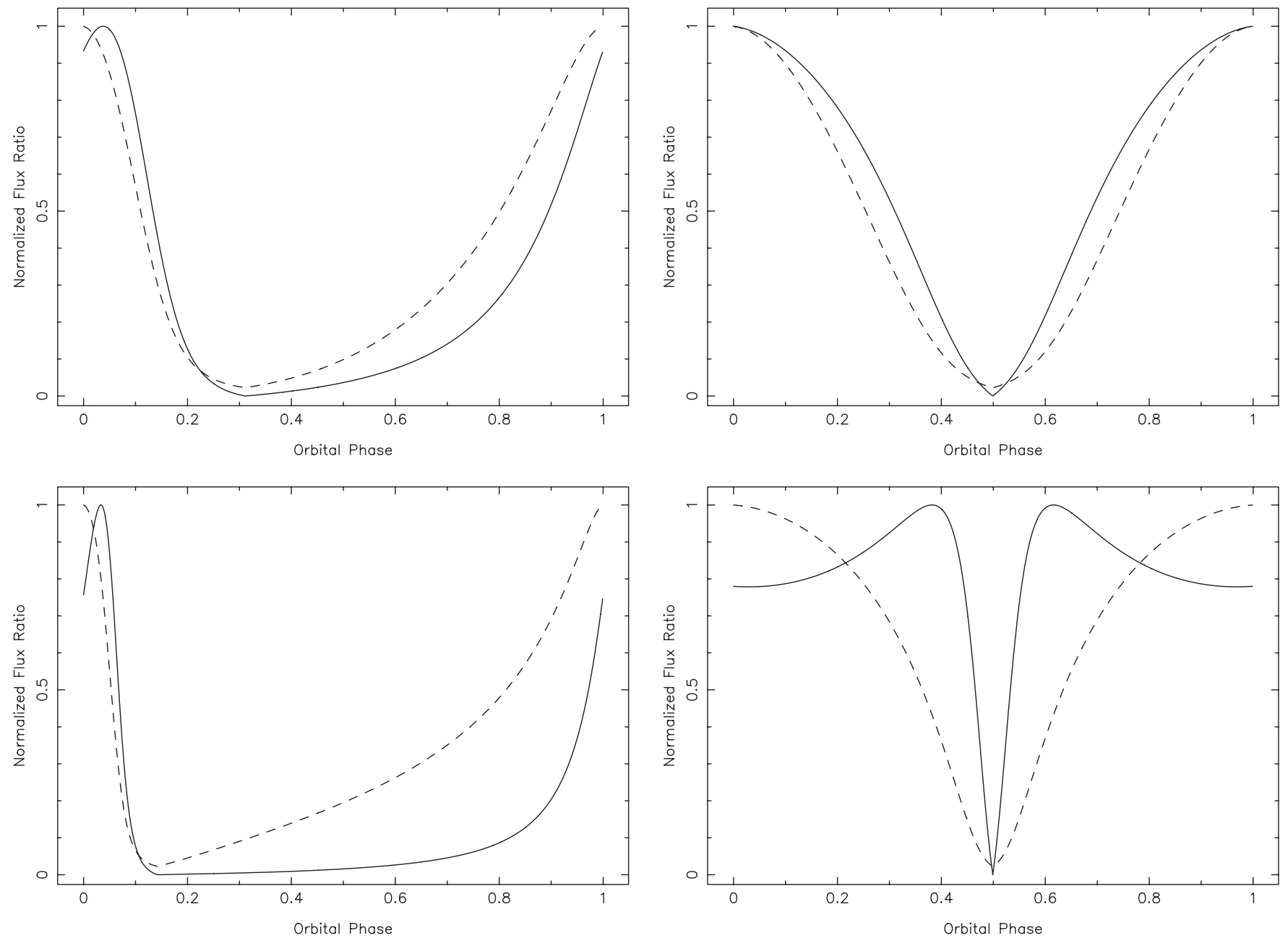

Figure 3. Phase functions (dashed line) and normalized flux ratios (solid line) for various eccentricities and periastron arguments; $e=0.3$ and $\omega=0^{\circ}$ (top left), $e=0.3$ and $\omega=90^{\circ}$ (top right), $e=0.6$ and $\omega=0^{\circ}$ (bottom left), $e=0.6$ and $\omega=90^{\circ}$ (bottom right).

\subsection{Resonance}

A number of systems have now been found to contain planets in eccentric orbits with some kind of resonant behavior. An interesting example is the HD 82943 planetary system (see Section 7.3), the 2:1 resonance of which has been studied in detail by Lee et al. (2006). The periodic simultaneous periastron passage of two planets produces a distinct signature from a phase amplitude perspective, although those moments will not necessarily be those of maximum flux ratio. In the context of radial velocity measurements, Giuppone et al. (2009) describe how resonant orbits can affect the detectability of exoplanets. Furthermore, it was shown by Anglada-Escudé et al. (2010) that 2:1 resonant systems can be misinterpreted as single-planet eccentric orbits when performing a fit to the radial velocity data. The same is true for phase curves of multi-planet systems, where resonant orbits can effectively hide the presence of the outer planet in the resulting phase curve since the combined phase variation will be periodic with time. In contrast, non-resonant planets will in general produce a non-periodic combined signal that will resolve as two separate phase functions with time.

Shown in Figure 4 are two example systems, each with two Jupiter radii planets. The orbits of the first system are in $2: 1$ resonance with $e=0.0$, while the planets of the second system are in $4: 1$ resonance with $e=0.5$. Given sufficient photometric precision and observing cadence, it may be possible to distinguish the deviant secondary peak of the first system and deduce the presence of the outer planet. However, this will be a difficult endeavor since the two peaks are relatively similar in amplitude. The second system presents an even more difficult problem, with the combination of high eccentricity and larger relative semimajor axis of the outer planet leading to a limited observation window, higher required cadence, and modest phase signature from the outer planet (seen close to an orbital phase of 0.95 in Figure 4). If one is unable to monitor this highest peak and also discern the difference in amplitude with the other three peaks then the presence of the outer planet will remain hidden to the observer. Thus, the derived system architecture based purely upon the phase variations will be incorrect. Resonant systems such as these currently comprise a small fraction of the total number of exoplanet systems. The relevance of this issue will increase as radial velocity surveys sample to longer periods and as Kepler discovers multi-planet systems, of which candidates have already been announced (Steffen et al. 2010).

\section{APPLICATION TO KNOWN EXOPLANETS}

Here, we apply the results of the previous sections to the known exoplanets. The orbital parameters of 370 planets were extracted using the Exoplanets Data Explorer. ${ }^{1}$ The data are

\footnotetext{
1 http://exoplanets.org/
} 

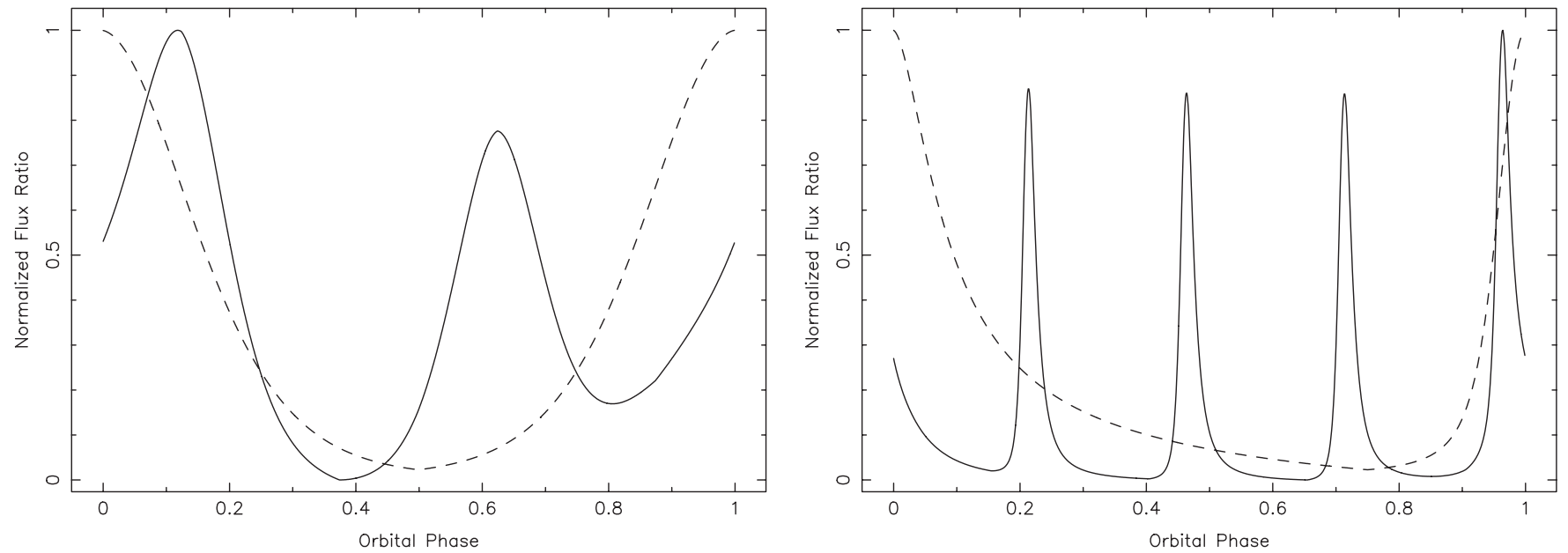

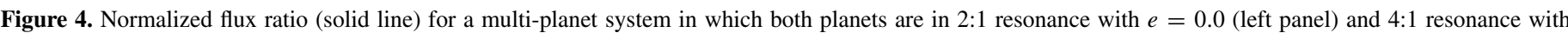
$e=0.5$ (right panel). The dashed line in each case represents the phase function of the outer planet.

current as of 2010 May 22. Since the flux ratio is $\propto R_{p}^{2}$, the unknown planetary radii for the non-transiting planets injects a degree of uncertainty into these calculations. The models of Bodenheimer et al. (2003) and Fortney et al. (2007) show that there is a clear planetary radius dependence upon stellar age as well as incident flux and planetary composition. However, Fortney et al. (2007) also showed that, for a given planetary composition, planetary radii should not vary substantially between orbital radii of 0.1-2.0 AU. Since most of the planets we are considering here lie beyond $0.1 \mathrm{AU}$ from their parent stars (by virtue of their eccentricity) and the mass distribution peaks at one Jupiter mass in this region, we fix the radius for each of the planets in this sample at 1 Jupiter radius, with the caveats mentioned above in mind.

As shown earlier, the orbital phase at which the maximum flux ratio, $\epsilon_{\max }$, occurs for an eccentric orbit depends upon the orbital orientation. Since Kepler's equation is a transcendental function, the integral of Equation (7) must be solved numerically in order to determine the maximum flux ratio for each planet. These calculations assume an orbital inclination of $i=90^{\circ}$ and thus the maximum flux ratios are upper limits in most cases, even though the radial velocity technique is biased toward detection of edge-on orbits since these produce larger radial velocity semi-amplitude signatures. We have also calculated the minimum time difference in units of orbital phase between where maximum flux occurs and where the flux drops to less than $5 \%$ of the difference between maximum and minimum flux. This quantity is designated $\Delta t$ and represents the minimum time over which observations of maximum effectiveness can be made.

These calculated values are plotted in Figure 5, both as a function of period and eccentricity. The value of $\Delta t$ is $\sim 0.37$ for all planets in circular orbits since this is where the phase difference for a simple cosine variation crosses the $<5 \%$ threshold described above. For eccentric orbits $\Delta t$ can be larger than expected, particularly where $\omega \sim 270^{\circ}$. Even so, the distribution shown in the top left panel of Figure 5 mirrors the distribution of orbital eccentricities. The bottom left panel shows that there is a minimum value of $\Delta t$ that may occur for a given eccentricity, but once again we see that this can float upward depending upon the value of $\omega$. The evident linear relation in log-space of the flux ratio on period shown in the top right panel demonstrates that the flux ratio is indeed dominated by the star-planet separation as one would expect. However, note the significant outliers beyond a period, $P$, of 200 days which are caused by the highly eccentric planets which pass through periastron close to a phase angle of $0^{\circ}$. Several of these systems are discussed in detail in Section 7.

The calculated values of $\epsilon_{\max }$ and $\Delta t$ for $\sim 70$ of the most eccentric known exoplanets are tabulated in Table 1. Of the planets represented in this table, the planet with the highest eccentricity, HD 80606b, is also the planet with the highest predicted flux ratio. This is not surprising considering that this planet's periastron passage is behind the star, leading to the high secondary eclipse probability and the subsequent observation of that eclipse by Laughlin et al. (2009). As described earlier and demonstrated by Figure 5, the flux ratios of the planets in Table 1 are dominated by the period and therefore the semimajor axis of the orbits.

\section{ORBITAL PARAMETER REFINEMENT}

As described by Kane et al. (2009), the refinement of orbital parameters is not only an essential component for successful detection of features which only appear for a small fraction of the orbit, it is also achievable with relatively few additional radial velocity measurements. This is particularly true of long-period planets whose orbits tend to have higher associated uncertainties and for which opportunities to observe at a particular place in the orbit are far less frequent.

For the goal of attempting to detect a planetary transit, it is the time of predicted transit mid-point which needs to be constrained. For optimal observations of phase variations, it is the time span during which the maximum change in flux ratio occurs which needs to be accurately determined, previously defined by the quantity $\Delta t$. The reason for this is because, even though the phase variation occurs over the entire orbit, it is assumed that the high cadence and precision needed will necessitate limited observing time using highly subscribed instruments (discussed further in Section 7).

Using the analogy of the transit window described by Kane et al. (2009), we introduce the concept of the phase prediction window, which is the time period during which a particular phase of the orbit could occur according to the uncertainties associated with the orbital period and the time of periastron passage. In Figure 6, we plot orbital eccentricity as a function of 

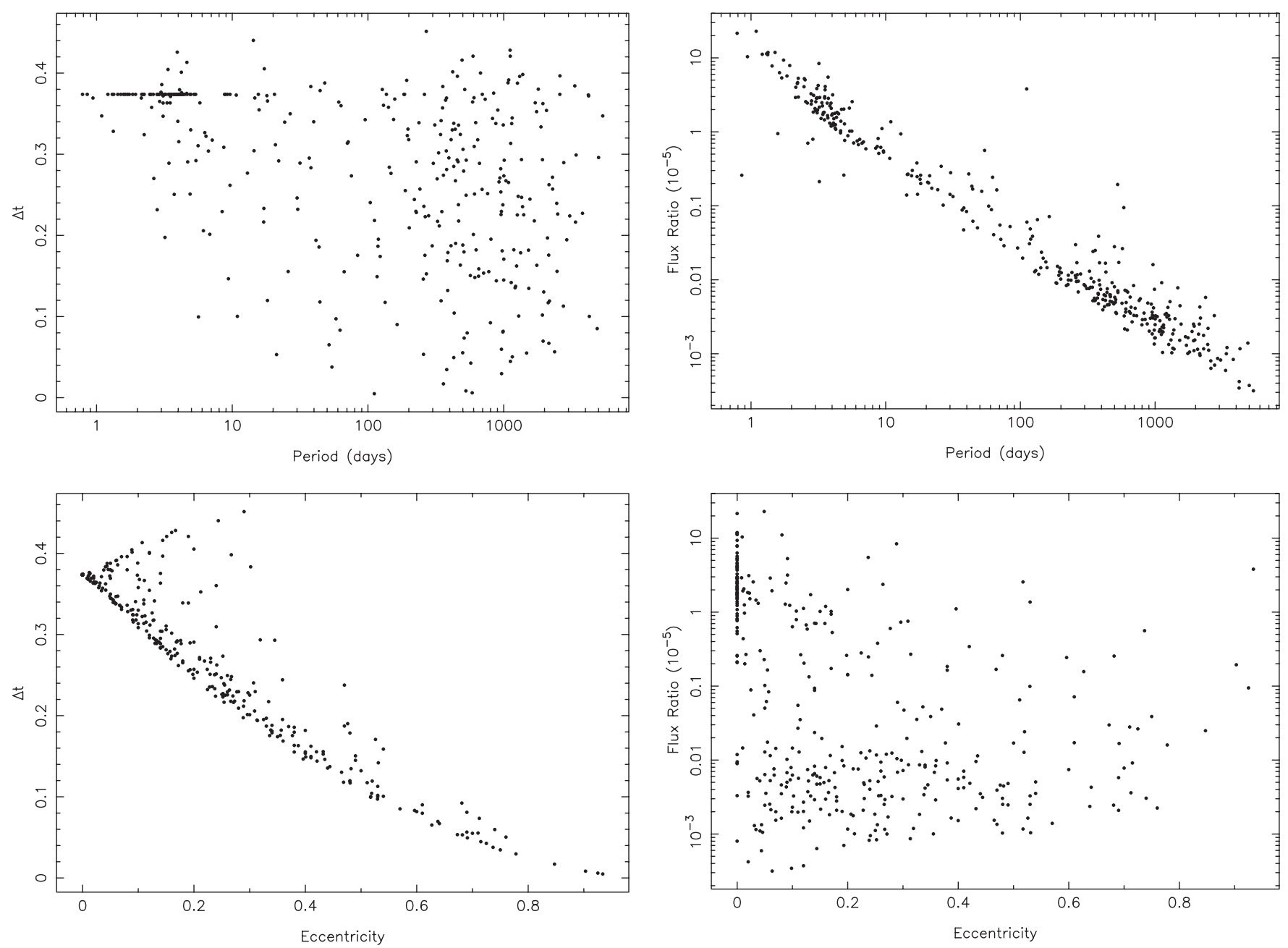

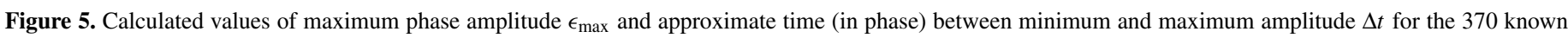

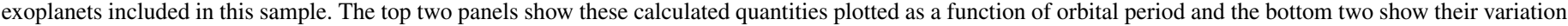
as a function of orbital eccentricity.

the phase window for the 370 exoplanets for which the necessary uncertainties were available (358). It is clear that the planets with the highest eccentricities are the most difficult cases to predict orbital phase locations. This is important because the most eccentric orbits tend to have much smaller $\Delta t$ values and so it is essential that orbital refinement be used to reduce the uncertainty in the phase prediction and hence the size of the associated window.

\section{ROBUSTNESS OF PHASE MODELS}

Although the phase function and albedo formulation adopted in this study to compute the expected phase variations are physically motivated, the sample of exoplanets with accurate measurements for these functions is relatively small. For example, the Hilton phase function (Equation (13)) is based upon the cloud maps of Venus and Jupiter which, although they produce similar phase functions, have their own unique cloud configurations with resulting slight variations in their respective phase functions. Likewise, real exoplanets could potentially exhibit a range of phase functions and albedo distributions beyond those considered here. As is clear from Equation (7), the flux ratio is linearly dependent on the geometric albedo. In other words, a $1 \%$ change in the value of $A_{g}$ translates into a $1 \%$ change in the value of $\epsilon_{\max }$. The geometric albedo has no effect on the time

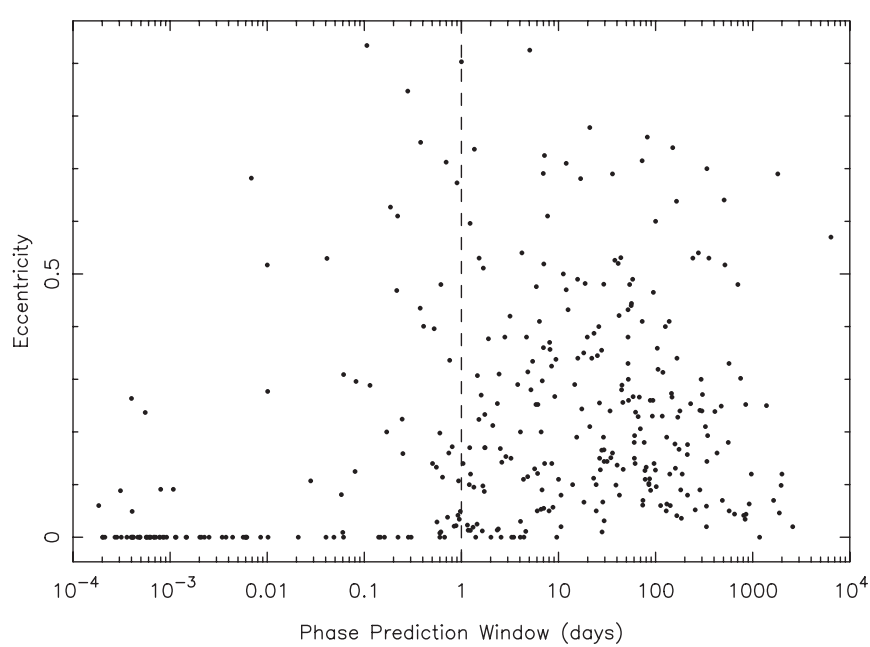

Figure 6. Orbital eccentricity as a function of phase prediction window for the known exoplanets. The dashed vertical line indicates a reasonable boundary beyond which observational attempts during a predicted phase location become difficult.

between minimum and maximum flux, $\Delta t$, since the shape of the phase variation is not altered.

A change in the phase function however, will alter both the calculated values of $\Delta t$ and $\epsilon_{\max }$. In order to quantify this effect, 
Table 1

$\epsilon_{\max }$ and $\Delta t$ for Eccentric Exoplanets

\begin{tabular}{|c|c|c|c|c|c|}
\hline Planet & $P$ (days) & $e$ & $\omega\left(^{\circ}\right)$ & $\Delta t$ & $\epsilon_{\max }\left(10^{-5}\right.$ \\
\hline HD 80606 b & 111.43 & 0.93 & 300.60 & 0.005 & 3.8029 \\
\hline HD 20782 b & 585.86 & 0.93 & 147.00 & 0.006 & 0.0946 \\
\hline HD $4113 \mathrm{~b}$ & 526.62 & 0.90 & 317.70 & 0.008 & 0.1939 \\
\hline HD 156846 b & 359.51 & 0.85 & 52.23 & 0.017 & 0.0249 \\
\hline HD $45350 \mathrm{~b}$ & 963.60 & 0.78 & 343.40 & 0.030 & 0.0160 \\
\hline HD 30562 b & 1157.00 & 0.76 & 81.00 & 0.050 & 0.0022 \\
\hline HD $20868 \mathrm{~b}$ & 380.85 & 0.75 & 356.20 & 0.035 & 0.0388 \\
\hline HD $41004 \mathrm{~A} \mathrm{~b}$ & 963.00 & 0.74 & 97.00 & 0.060 & 0.0030 \\
\hline HD $37605 \mathrm{~b}$ & 54.23 & 0.74 & 211.60 & 0.038 & 0.5608 \\
\hline HD $222582 \mathrm{~b}$ & 572.38 & 0.73 & 319.01 & 0.043 & 0.0263 \\
\hline HD 2039 b & 1120.00 & 0.71 & 344.10 & 0.045 & 0.0091 \\
\hline iota Dra b & 511.10 & 0.71 & 91.58 & 0.073 & 0.0036 \\
\hline HD $96167 \mathrm{~b}$ & 498.90 & 0.71 & 285.00 & 0.055 & 0.0281 \\
\hline HD 86264 b & 1475.00 & 0.70 & 306.00 & 0.055 & 0.0078 \\
\hline HAT-P-13 c & 428.50 & 0.69 & 176.70 & 0.050 & 0.0167 \\
\hline HD 159868 b & 986.00 & 0.69 & 97.00 & 0.081 & 0.0021 \\
\hline HD $43848 \mathrm{~b}$ & 2371.00 & 0.69 & 229.00 & 0.056 & 0.0058 \\
\hline HD $17156 b$ & 21.22 & 0.68 & 121.90 & 0.053 & 0.2549 \\
\hline 16 Cyg B b & 798.50 & 0.68 & 85.80 & 0.092 & 0.0025 \\
\hline HD 89744 b & 256.78 & 0.67 & 195.10 & 0.053 & 0.0298 \\
\hline HD $39091 \mathrm{~b}$ & 2151.00 & 0.64 & 330.24 & 0.067 & 0.0043 \\
\hline HD 131664 b & 1951.00 & 0.64 & 149.70 & 0.070 & 0.0024 \\
\hline HD $74156 b$ & 51.65 & 0.63 & 176.50 & 0.065 & 0.1567 \\
\hline HD $171028 b$ & 538.00 & 0.61 & 305.00 & 0.080 & 0.0172 \\
\hline HD 154672 b & 163.94 & 0.61 & 265.00 & 0.090 & 0.0715 \\
\hline HD $16175 b$ & 990.00 & 0.60 & 222.00 & 0.082 & 0.0074 \\
\hline HD $3651 \mathrm{~b}$ & 62.22 & 0.60 & 245.50 & 0.083 & 0.2431 \\
\hline HD 190984 b & 4885.00 & 0.57 & 318.00 & 0.085 & 0.0014 \\
\hline HIP 2247 b & 655.60 & 0.54 & 112.20 & 0.159 & 0.0035 \\
\hline HD $175167 \mathrm{~b}$ & 1290.00 & 0.54 & 325.00 & 0.101 & 0.0051 \\
\hline HD 190228 b & 1136.10 & 0.53 & 101.20 & 0.142 & 0.0010 \\
\hline HD $87883 b$ & 2754.00 & 0.53 & 291.00 & 0.113 & 0.0033 \\
\hline HD 142022 b & 1928.00 & 0.53 & 170.00 & 0.102 & 0.0025 \\
\hline HD 108147 b & 10.90 & 0.53 & 308.00 & 0.100 & 1.3664 \\
\hline HD 168443 b & 58.11 & 0.53 & 172.95 & 0.097 & 0.0988 \\
\hline HD $81040 \mathrm{~b}$ & 1001.70 & 0.53 & 81.30 & 0.171 & 0.0016 \\
\hline HIP 5158 b & 345.72 & 0.52 & 252.00 & 0.119 & 0.0241 \\
\hline HD 4203 b & 431.88 & 0.52 & 329.10 & 0.104 & 0.0127 \\
\hline HD $217107 \mathrm{c}$ & 4270.00 & 0.52 & 198.60 & 0.100 & 0.0012 \\
\hline HAT-P-2 b & 5.63 & 0.52 & 185.22 & 0.100 & 2.5595 \\
\hline HD $1237 b$ & 133.71 & 0.51 & 290.70 & 0.117 & 0.0650 \\
\hline HD $142415 b$ & 386.30 & 0.50 & 255.00 & 0.132 & 0.0170 \\
\hline HD 34445 b & 1000.00 & 0.49 & 131.00 & 0.145 & 0.0025 \\
\hline HD $215497 \mathrm{c}$ & 567.94 & 0.49 & 45.00 & 0.150 & 0.0048 \\
\hline HD 106252 b & 1531.00 & 0.48 & 292.80 & 0.135 & 0.0045 \\
\hline HD $33636 b$ & 2127.70 & 0.48 & 339.50 & 0.117 & 0.0025 \\
\hline HD $33283 b$ & 18.18 & 0.48 & 155.80 & 0.120 & 0.2591 \\
\hline HD 196885 b & 1333.00 & 0.48 & 78.00 & 0.179 & 0.0010 \\
\hline HD $181433 \mathrm{~d}$ & 2172.00 & 0.48 & 330.00 & 0.119 & 0.0030 \\
\hline HD $210277 \mathrm{~b}$ & 442.19 & 0.48 & 119.10 & 0.190 & 0.0046 \\
\hline HD $154857 \mathrm{~b}$ & 409.00 & 0.47 & 59.00 & 0.187 & 0.0039 \\
\hline HD 187085 b & 986.00 & 0.47 & 94.00 & 0.238 & 0.0014 \\
\hline HD 147018 b & 44.24 & 0.47 & 335.97 & 0.118 & 0.1681 \\
\hline HD $66428 \mathrm{~b}$ & 1973.00 & 0.47 & 152.90 & 0.130 & 0.0015 \\
\hline HD 50554 b & 1224.00 & 0.44 & 7.40 & 0.137 & 0.0031 \\
\hline HD $23127 \mathrm{~b}$ & 1214.00 & 0.44 & 190.00 & 0.135 & 0.0035 \\
\hline HD 202206 b & 255.87 & 0.44 & 161.18 & 0.146 & 0.0114 \\
\hline HD $74156 \mathrm{c}$ & 2473.00 & 0.43 & 258.60 & 0.156 & 0.0022 \\
\hline $4 \mathrm{UMa}$ b & 269.30 & 0.43 & 23.81 & 0.153 & 0.0096 \\
\hline HD $213240 \mathrm{~b}$ & 882.70 & 0.42 & 201.00 & 0.144 & 0.0048 \\
\hline HD 117618 b & 25.83 & 0.42 & 254.00 & 0.156 & 0.3417 \\
\hline HD 141937 b & 653.22 & 0.41 & 187.72 & 0.150 & 0.0058 \\
\hline HD $65216 b$ & 613.10 & 0.41 & 198.00 & 0.148 & 0.0071 \\
\hline HD $126614 \mathrm{~A} \mathrm{~b}$ & 1244.00 & 0.41 & 243.00 & 0.162 & 0.0042 \\
\hline 70 Vir b & 116.69 & 0.40 & 358.71 & 0.149 & 0.0307 \\
\hline HD $171238 b$ & 1523.00 & 0.40 & 47.00 & 0.182 & 0.0015 \\
\hline
\end{tabular}

Table 1

(Continued)

\begin{tabular}{lrcccc}
\hline \hline \multicolumn{1}{c}{ Planet } & $P$ (days) & $e$ & $\omega\left(^{\circ}\right)$ & \multicolumn{1}{c}{$\Delta t$} & $\epsilon_{\max }\left(10^{-5}\right)$ \\
\hline HD 5388 b & 777.00 & 0.40 & 324.00 & 0.155 & 0.0055 \\
HD 11977 b & 711.00 & 0.40 & 351.50 & 0.154 & 0.0041 \\
HD 181433 b & 9.37 & 0.40 & 202.00 & 0.147 & 1.1056 \\
14 Her b & 1754.00 & 0.39 & 19.60 & 0.163 & 0.0016 \\
HD 125612 b & 510.00 & 0.38 & 21.00 & 0.180 & 0.0054 \\
42 Dra b & 479.10 & 0.38 & 218.70 & 0.163 & 0.0091 \\
\hline
\end{tabular}

Note. $\Delta t$ in units of orbital phase.

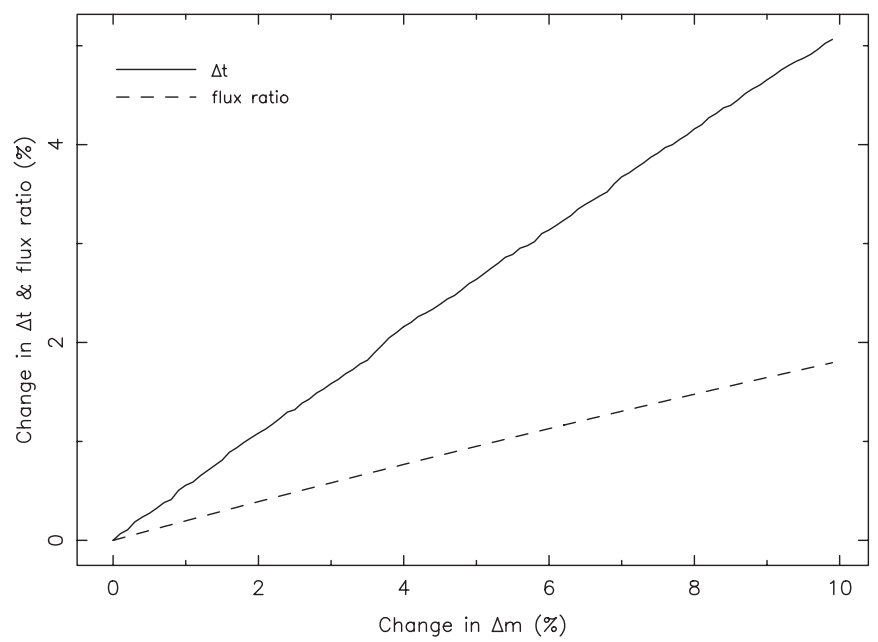

Figure 7. Percentage change in the values of $\Delta t$ and $\epsilon_{\max }$ as a function the percentage variation in the planetary visual magnitude correction (see Equation (12)) for the planets in Table 1.

we varied the value of the planetary visual magnitude correction, $\Delta m$, as described in Equation (12). This was performed for all of the planets shown in Table 1, from which the the mean of the percentage change in $\Delta t$ and $\epsilon_{\max }$ was calculated for those planets. The results of this simulation are shown in Figure 7. Even a substantial change in the phase function of $10 \%$ leads to a relatively minor change in the expected value of $\Delta t$ and an even smaller impact on the expected flux ratio. Additionally, the magnitude of these changes are largest for eccentric planets which are more sensitive to the location of the peaks in the phase curve.

\section{CASE STUDIES AND DETECTABILITY}

Here we present specific examples of predicted phase amplitudes and detectability for several of the known exoplanets. In evaluating whether these signatures are detectable or if instead calculating these signatures will remain a theoretical exercise for the immediate future, consider the precision of the Hubble Space Telescope (HST) and the MOST satellite. Both of these telescopes have observed the $V=7.65$ star HD 209458. The HST observations by Brown et al. (2001) achieved a precision of $1.1 \times 10^{-4}$ and the MOST observations by Croll et al. (2007) achieved a precision of $3.5 \times 10^{-3}$. In these cases, the necessarily high cadence resulting from the brightness of the host stars could be used to advantage by binning the data to produce higher precision. In addition, the ellipsoidal variations detected by Welsh et al. (2010) using Kepler data are of amplitude $3.7 \times 10^{-5}$. 


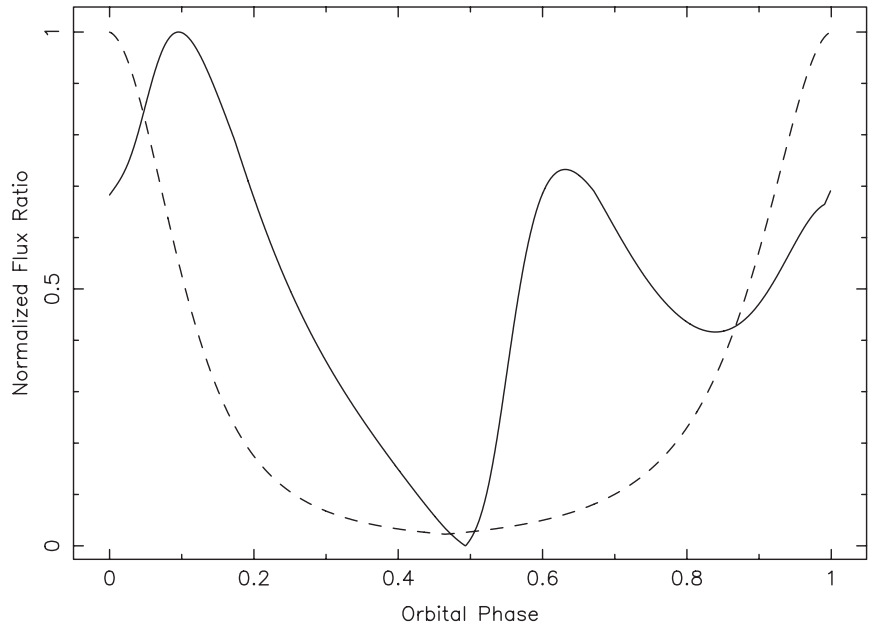

Figure 8. Predicted normalized flux ratio (solid line) for the HD 82943 system, the planets of which are in 2:1 resonance. The dashed line represents the phase function for the outer planet.

\subsection{The HD 37605 System}

Our current knowledge of the HD 37605 system consists of a single planet in a $\sim 54$ day, highly eccentric orbit (Cochran et al. 2004). This places this planet close to the top of the list shown in Table 1. The peak flux ratio from this planet is expected to be $0.56 \times 10^{-5}$, more than a factor of 10 smaller than that for HAT-P-7b. Even so, the peak flux ratio is helped substantially by the periastron argument of $\omega=211^{\circ}$ which places the periastron passage close to the observer-star line of sight on the far side of the star.

The minimum time between minimum and maximum flux ratio is 0.04 of the orbital phase or $\sim 2.0$ days. Conversely, the phase prediction window for this planet at the time of discovery (2004) was $\sim 1.4$ days. At the time of writing, this window has since grown to $\sim 19$ days due to the $\sim 40$ orbits which have occurred since then. Thus, the precision of the orbital parameters require further improvement before a robust attempt to only observe the $\Delta t$ section of the orbital phase is made.

\subsection{The HAT-P-13 System}

The HAT-P-13 system (Bakos et al. 2009) is particularly interesting because it consists of an inner transiting planet $(P=2.92$ days $)$ in a circular orbit with an outer companion in a long-period ( $P=428.5$ days) eccentric orbit. Additional data acquired by Winn et al. (2010) provide evidence for a third body in the system. The coplanarity of the system remains in question however since the refined orbital parameters for the outer planet have not yet resulted in a transit detection. Could this be resolved with observations during the predicted maximum flux ratio occurrence of the outer planet? This will be difficult for the following reasons. First, the maximum flux ratio of the inner planet is predicted to be $3.11 \times 10^{-5}$ compared to $0.017 \times 10^{-5}$ for the outer planet. Detecting the signature of the outer planet would be a challenging detection task even if the signature of the inner planet were not present. Second, since the inclination of the outer planet is not known, the predicted flux ratio becomes an upper limit, potentially making the detection criteria even more dire. The substantiative dominance of the inner planet over the phase signature makes this far too challenging a task for any current instruments.

\subsection{The HD 82943 System}

The HD 82943 system contains two planets in eccentric orbits in a well-studied 2:1 resonance (Lee et al. 2006). The inner and outer planets have periods of 219 and 441 days respectively but have near identical maximum flux ratios of $\sim 0.008 \times 10^{-5}$ well beyond the detection limits of current instruments. The reason for the similarity in the peak flux ratios is due to the orbital elements in the system. The eccentricity of the orbits combined with the resonance has led to the axes of the orbits being almost $\pi$ out of phase with each other. This leads to the complex structure for the total flux ratio variation of the system, shown in Figure 8. Note that the periastron passage of the outer planet occurs behind the star, whereas it occurs in front of the star for the inner planet, yielding a relative increase in the maximum flux ratio for the outer planet, partially compensating for the larger semimajor axis.

\section{CONCLUSIONS}

Current generation space missions are already detecting exoplanet phase variations in the optical (e.g., Kepler) and the IR (e.g., Spitzer). The steps these produce toward characterizing the atmospheres of these exoplanets are significant since they provide direct measurements of the atmospheric albedo and thermal properties. This has currently been primarily undertaken for short-period planets since many of these transit and produce phase variations on easily observable timescales. However, current radial velocity surveys are biased toward planets whose orbits are closer to edge-on, since larger semi-amplitude signatures are produced, and therefore biased toward planets with larger predicted phase amplitudes.

We have shown here how time- and position-dependent functions for the geometric albedo and phase can be used to describe expected phase variations for long-period eccentric giant planets. There is a clear degeneracy with orbital inclination and resonance when considering multi-planet systems and care must be taken to account for these possibilities. Applying these results to the known exoplanets shows that many longperiod eccentric planets can have significant peak flux ratios, comparable to those of short-period planets. Additionally, the phase prediction windows of eccentric planets, during which observations will be optimally placed, will tend to be poorly constrained. The refinement of orbital parameters for the known exoplanets is clearly a key component for optimal observations of the eccentric planets during maximum phase amplitude. Improving the measured orbits of long-period planets is already being undertaken by such projects as the Transit Ephemeris Refinement and Monitoring Survey (TERMS; Kane et al. 2009). However, most of the predicted flux ratios for the known planets push heavily against the boundaries of what is achievable with current ground and space-based instruments. A thorough search of all these planets will therefore likely need to await future generation telescopes, such as the European Extremely Large Telescope (E-ELT), the Thirty Meter Telescope (TMT), the Giant Magellan Telescope (GMT), and the James Webb Space Telescope (JWST).

As more science results are released by the Kepler mission, the study of photometric phase variations of long-period planets will become increasingly relevant. Not only is it an existing mission which has already detected phase variations in the light curve of HAT-P-7b, it is specifically looking for transiting planets where there is an a priori knowledge that the orbital inclination is favorable toward maximum phase amplitude. 
Additionally, Kepler will eventually detect transiting longperiod $(P>100$ days) planets where the bias will certainly be toward eccentric orbits since those have a higher probability of transiting (Kane \& von Braun 2008). These future discoveries will be prime candidates to detect the phase variations described here.

The authors thank David Ciardi for several useful discussions. We also thank the anonymous referee whose comments greatly improved the quality of the paper. This research has made use of the Exoplanet Orbit Database and the Exoplanet Data Explorer at http://exoplanets.org.

\section{REFERENCES}

Anglada-Escudé, G., López-Morales, M., \& Chambers, J. E. 2010, ApJ, 709, 168

Bakos, G. Á., et al. 2009, ApJ, 707, 446

Barbieri, M., et al. 2007, A\&A, 476, L13

Bodenheimer, P., Laughlin, G., \& Lin, D. N. C. 2003, ApJ, 592, 555

Brown, T. M., Charbonneau, D., Gilliland, R. L., Noyes, R. W., \& Burrows, A. 2001, ApJ, 552, 699

Burrows, A., Ibgui, L., \& Hubeny, I. 2008, ApJ, 682, 1277

Butler, R. P., Marcy, G. W., Fischer, D. A., Brown, T. M., Contos, A. R., Korzennik, S. G., Nisenson, P., \& Noyes, R. W. 1999, ApJ, 526, 916

Cahoy, K. L., Marley, M. S., \& Fortney, J. J. 2010, ApJ, in press (arXiv:1009.3071)

Charbonneau, D., Noyes, R. W., Korzennik, S. G., Nisenson, P., Jha, S., Vogt, S. S., \& Kibrick, R. I. 1999, ApJ, 522, L145

Cochran, W. D., et al. 2004, ApJ, 611, L133

Collier Cameron, A., Horne, K., Penny, A., \& Leigh, C. 2002, MNRAS, 330, 187

Cowan, N. B., \& Agol, E. 2008, ApJ, 678, L129

Cowan, N. B., Agol, E., \& Charbonneau, D. 2007, MNRAS, 379, 641

Croll, B., et al. 2007, ApJ, 658, 1328
Dyudina, U. A., Sackett, P. D., Bayliss, D. D. R., Seager, S., Porco, C. C., Throop, H. B., \& Dones, L. 2005, ApJ, 618, 973

Fortney, J. J., Marley, M. S., \& Barnes, J. W. 2007, ApJ, 659, 1661

Gelino, D. M., Balman, S., Kiziloğlu, Ü., Yilmaz, A., Kalemci, E., \& Tomsick, J. A. 2006, ApJ, 642, 438

Giuppone, C. A., Tadeu dos Sanots, M., Beauǵe, C., Ferraz-Mello, S., \& Michtchenko, T. A. 2009, ApJ, 699, 1321

Harrington, J., Hansen, B. M., Luszcz, S. H., Seager, S., Deming, D., Menou, K., Cho, J. Y.-K., \& Richardson, L. J. 2006, Science, 314, 623

Hilton, J. L. 1992, in Explanatory Supplement to the Astronomical Almanac, ed. P. K. Seidelmann (Mill Valley, CA: Univ. Science Books), 383

Iro, N., \& Deming, L. D. 2010, ApJ, 712, 218

Kane, S. R., Mahadevan, S., von Braun, K., Laughlin, G., \& Ciardi, D. R. 2009, PASP, 121, 1386

Kane, S. R., \& von Braun, K. 2008, ApJ, 689, 492

Kane, S. R., \& von Braun, K. 2009, PASP, 121, 1096

Knutson, H. A., Charbonneau, D., Cowan, N. B., Fortney, J. J., Showman, A. P., Agol, E., \& Henry, G. W. 2009a, ApJ, 703, 769

Knutson, H. A., et al. 2009b, ApJ, 690, 822

Laughlin, G., Deming, D., Langton, J., Kasen, D., Vogt, S., Butler, P., Rivera, E., \& Meschiari, S. 2009, Nature, 457, 562

Lee, M. H., Butler, R. P., Fischer, D. A., Marcy, G. W., \& Vogt, S. S. 2006, ApJ, 641,1178

Leigh, C., Collier Cameron, A., Horne, K., Penny, A., \& James, D. 2003, MNRAS, 344, 1271

Mallama, A. 2009, Icarus, 204, 11

McArthur, B. E., Benedict, G. F., Barnes, R., Martioli, E., Korzennik, S., Nelan, E., \& Butler, R. P. 2010, ApJ, 715, 1203

Rodler, F., Kürster, M., \& Henning, T. 2008, A\&A, 485, 859

Rodler, F., Kürster, M., \& Henning, T. 2010, A\&A, 514, A23

Rowe, J. F., et al. 2008, ApJ, 689, 1345

Snellen, I. A. G., de Mooij, E. J. W., \& Albrecht, S. 2009, Nature, 459, 543

Steffen, J. H., et al. 2010, ApJ, in press (arXiv:1006.2763)

Sudarsky, D., Burrows, A., Hubeny, I., \& Li, A. 2005, ApJ, 627, 520

Sudarsky, D., Burrows, A., \& Pinto, P. 2000, ApJ, 538, 885

Welsh, W. F., Orosz, J. A., Seager, S., Fortney, J. J., Jenkins, J., Rowe, J. F., Koch, D., \& Borucki, W. J. 2010, ApJ, 713, L145

Winn, J. N., et al. 2010, ApJ, 718, 575 